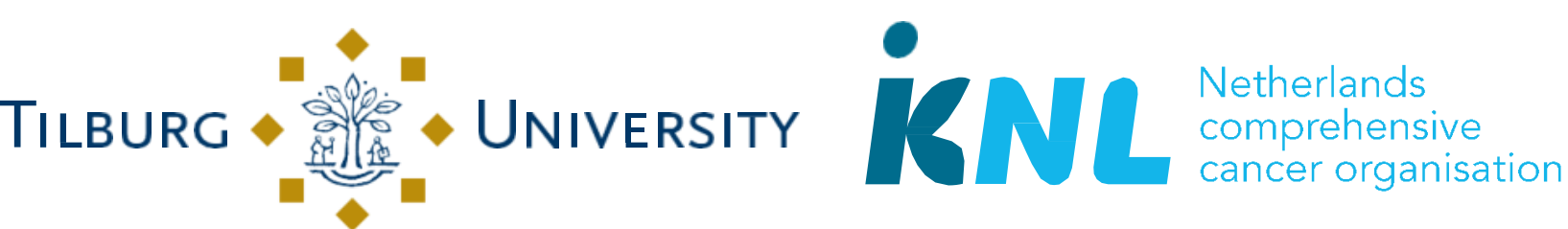

\section{Do people improve health behavior after their partner is diagnosed with cancer? A Danish cohort study}

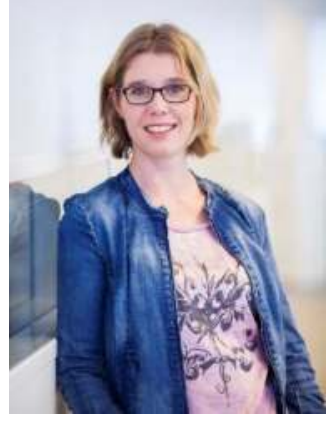

Background

The cancer diagnosis might evoke behavioral change among partners of cancer patients.

\section{This study aims to:}

Assess if partners of cancer patients make more health behavior changes compared to persons whose partner remained cancerfree.

\section{Methods}

- Nested in the Danish Diet, Cancer and Health study - a prospective cohort study

- 672 couples where one person was diagnosed with cancer and 5534 couples who remained cancer-free (Fig. 1)

- Questionnaires assessing diet, physical activity, smoking, alcohol and BMI were administered in 1993-1997 and 2000-2002.

- Logistic regression analyses to assess health behavior change among partners of cancer patients, and for the two subgroups

1 partners of patients who died after cancer and

2 partners of patients who remained alive after cancer

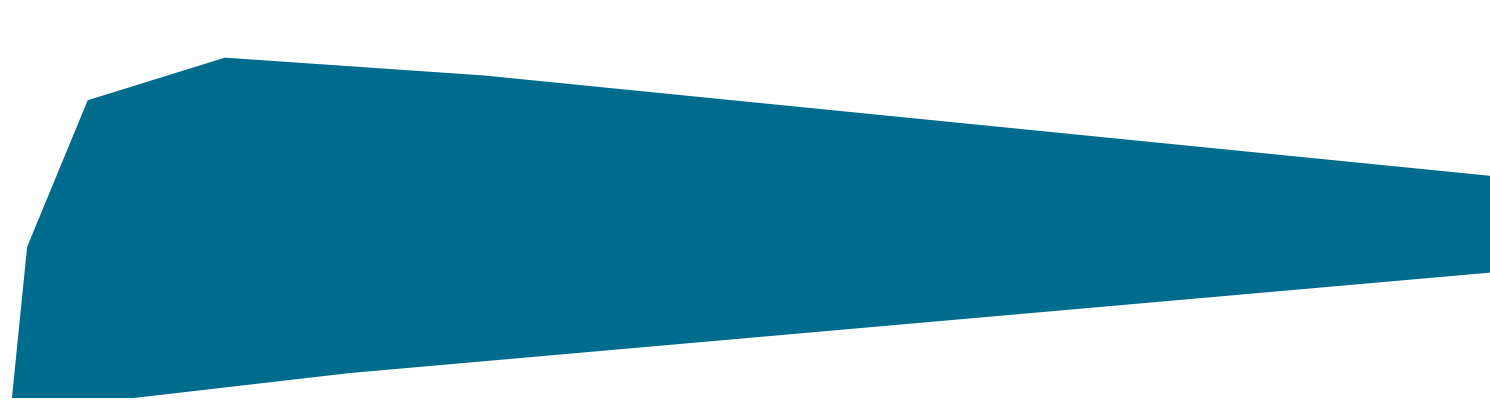

Smoking

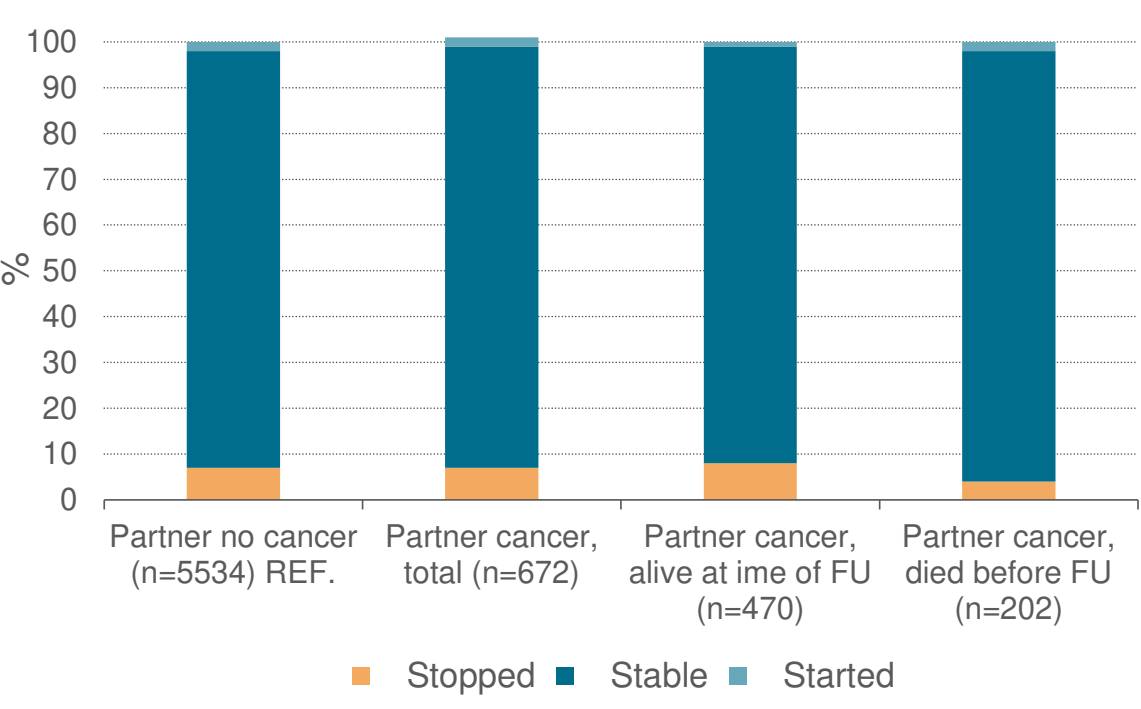

Alcohol intake

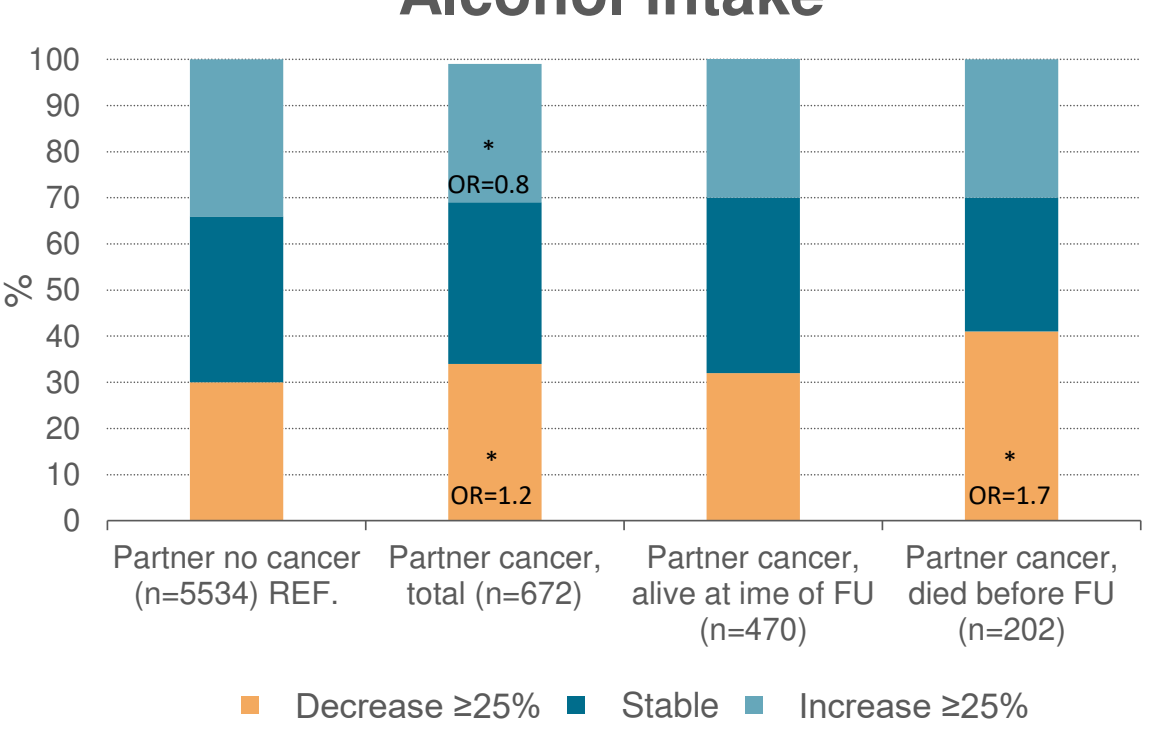

- Decrease $\geq 25 \%$ - Stable In Increase $\geq 25 \%$

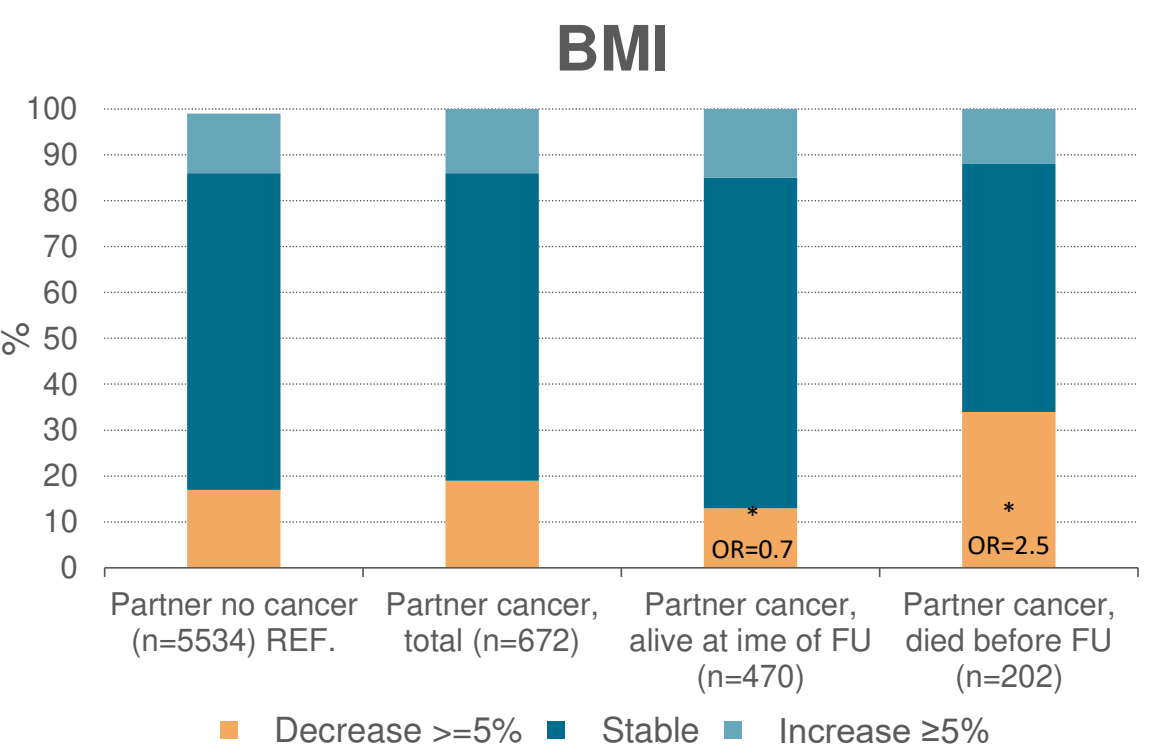

Fruit intake

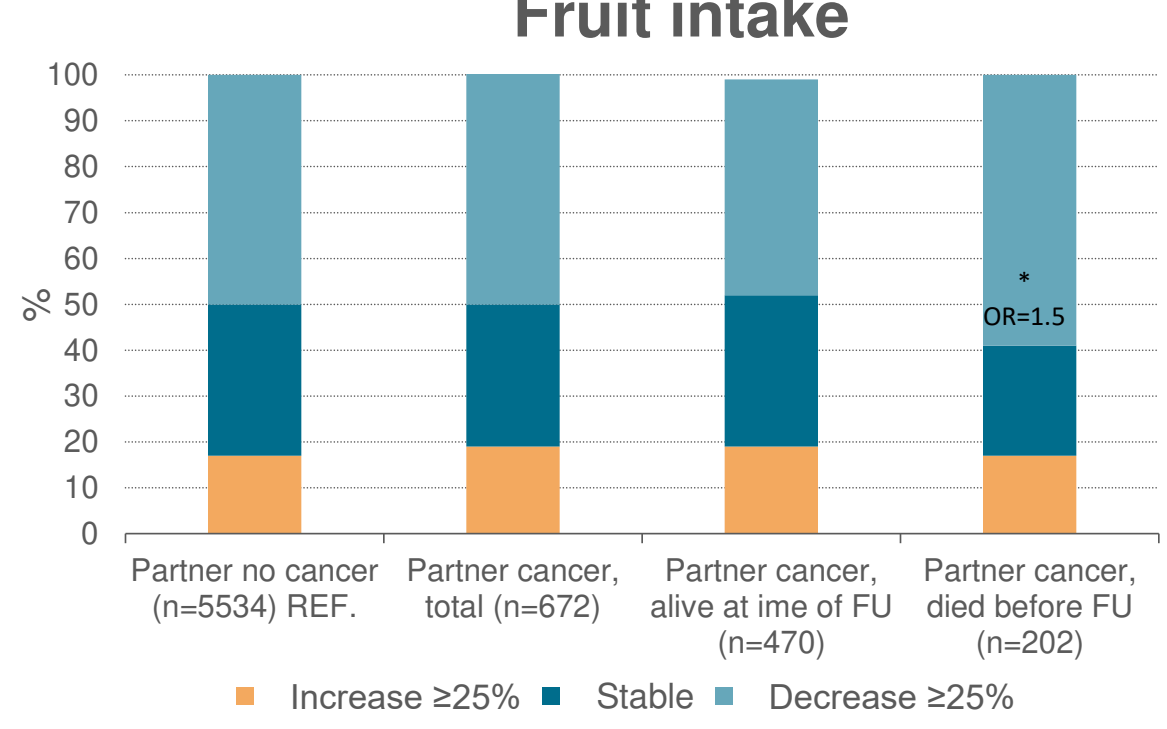

\section{Results}

- Mean age 55 years; $55 \%$ women

- In general, partners of cancer patients were more likely to decrease alcohol intake (Fig. 2).

- Among the subgroups, partners of persons who remained alive were less likely to decrease BMI, whereas bereaved partners were more likely to decrease BMI, and alcohol and fruit intake, and to increase sugared beverages (Fig. 2).

- Men tended to improve and women to worsen their physical activity following the cancer diagnosis of their partner.

\section{Conclusions}

- Partners of cancer patients show limited changes in health behaviors after the cancer diagnosis, but bereaved partners tend to worsen health behaviors after the patient's death.

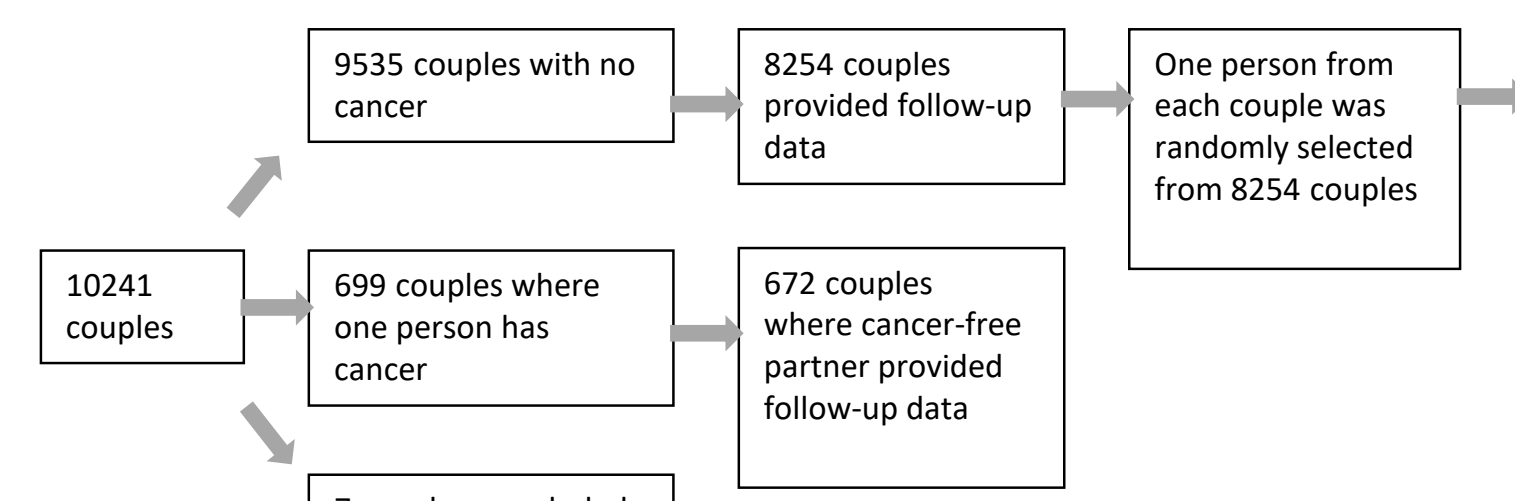
age groups and gender to the partner without cancer using the smallest ratio $(1: 8)$ 5534 persons
7 couples - excluded due to both person
having cancer

Fig. 1 Selection process of study participants

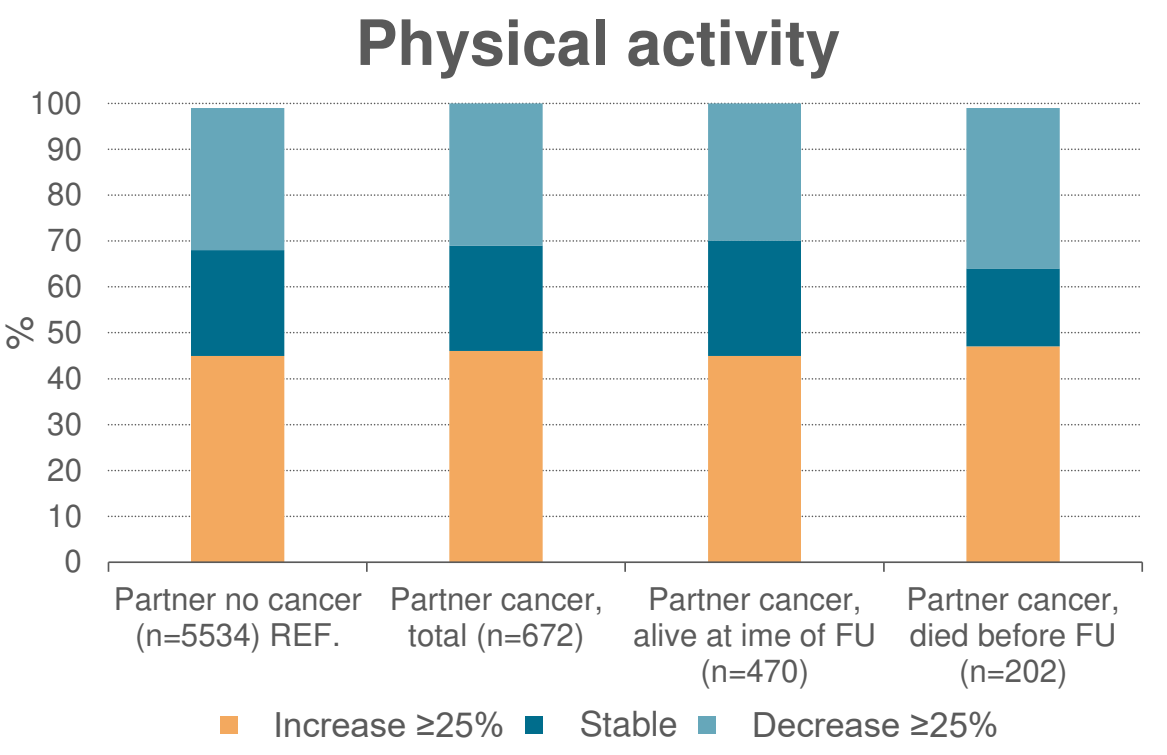

Sugar-sweetened beverages

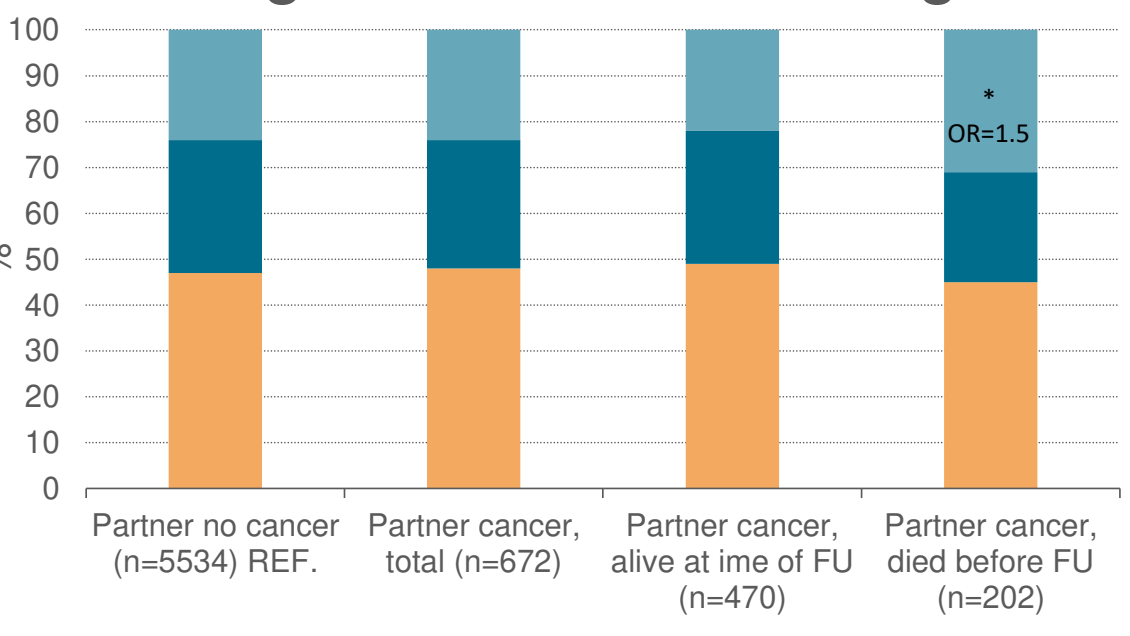

- Decrease $\geq 25 \%$ - Stable a Increase $\geq 25 \%$

Fig.2 Proportion of partners who changed their health behavior according to cancer status.

* indicates $p<0.05$; OR=Odds Ratio for improving (orange) vs. not improving (stable or worsening) the health behavior or for worsening (light blue) vs. not worsening (stable or improving). Partners without cancer are the reference group. 